Article

\title{
Characterization of the Active Constituents in Shixiao San Using Bioactivity Evaluation Followed by UPLC-QTOF and Markerlynx Analysis
}

\author{
Wei Zhou, Shu-Lan Su *, Jin-Ao Duan *, Jian-Ming Guo, Da-Wei Qian, Er-Xin Shang and \\ Jin Zhang
}

Jiangsu Key laboratory for TCM Formulae Research (Supported by the Cyrus Tang Foundation of USA); Nanjing University of Chinese Medicine, Nanjing 210046, China;

E-Mail: zhouwei19840@163.com (W.Z.)

* Authors to whom correspondence should be addressed; E-Mails: sushulan1974@163.com (S.-L.S.); duanja@163.com (J.A.D.); Tel.: + 8625 85811116; Fax: + 862585811916.

Received: 28 April 2010; in revised form: 16 August 2010 / Accepted: 19 August 2010 /

Published: 3 September 2010

\begin{abstract}
Shixiao San is a famous Traditional Chinese Medicine (TCM) formula that has been used for a long time for the treatment of gynecological diseases. In this paper, the active constituents in Shixiao San were characterized by using bioactivity evaluation followed by UPLC-QTOF and Markerlynx ${ }^{\mathrm{TM}}$ analysis. The analgesic activities of two extracts of Shixiao San were evaluated using the hot-plate test, acetic acid-induced writhing and dysmenorrhea mice model. The results showed that the analgesic activity of Shixiao San vinegary extract (boiling vinegar) was superior to the aqueous extract. UPLCQTOF and Markerlynx ${ }^{\mathrm{TM}}$ analytic results showed that the process of boiling in vinegar may improve the dissolution of Shixiao San flavanoids, and these flavanoids may contribute to the observed analgesic activity. This work demonstrated that UPLC/QTOFMS and Markerlynx ${ }^{\mathrm{TM}}$ could serve as new methods for fast generation and automated analysis of information-rich data from Chinese herbal medicines.
\end{abstract}

Keywords: Shixiao San; analgesic activity; active components; UPLC-QTOF-MS; Markerlynx $^{\mathrm{TM}}$ 


\section{Introduction}

Shixiao San has analgesic activity and can dissipate blood stasis and it has been widely used in the clinic for centuries to treat gynecological disease [1]. Shixiao San consists of two crude herbs, Pollen Typha (Pollen of Typha angustifolia L.) named "Puhuang" in China, and Faeces Trogopterori (faeces of the complex-toothed flying squirrel Trogopterus xanthipes Milne-Edwards), named "Wulingzhi" in China.

It has been recorded in ancient documents like the Complete Collection of Prescriptions for Women that Shixiao San is first boiled with vinegar, and then extracted by water (Vinegary Extract). In addition, Shixiao San is clinically used after water extraction (Aqueous Extract) to treat many diseases [2]. The aqueous extract of Shixiao San has been reported to have multiple bio-activities, such as analgesia, sedation, anti-atherosclerotic, anti-inflammatory, and so on. [3,4]. The aqueous extract of Shixiao San was also reported to contain flavonoids, organic acids, esters of fatty acids, etc. [3,5], but until now the bioactivity and the chemical components of the vinegary extract of Shixiao San have not been reported. Moreover, there have been no studies of the differences in bio-activity and chemical components between the two Shixiao San preparations, therefore, in order to provide a scientific explanation and basis for clinical application, it is necessary to study and compare the bioactivity as well as the chemical constitution of the two extracts of Shixiao San.

It is well accepted that herbal medicines exert their efficacies on multi-targets through multicomponents [6], and one or a few marker compound(s) cannot provide holistic information about a combinatorial formula [7]. However, due to the complexity of the chemical compositions of TCMs, the bioactive compounds and the therapeutic mechanisms of most TCMs are still unknown [8]. Therefore, new methods were needed to analyze the multi-components and their contribution to the efficacy of TCMs.

Ultra performance liquid chromatography (UPLC) coupled with a photodiode array detector (PDA) and time-of-flight mass spectrometry (TOFMS) is a newly developed hyphenated technique (UPLCPDA-TOFMS) [7]. It can provide a huge amount of information more rapidly and efficiently than other techniques. High selectivity and sensitivity have allowed the wide application of UPLC-MS/MS for quantitative and qualitative analysis, as well as metabolite analysis and identification from bioassays of complex samples such as TCMs [9]. To analyze and compare the information-rich spectroscopic data generated by UPLC/MS analysis from different samples, the commercial software Markerlynx ${ }^{\mathrm{TM}}$ has been used to process the complex data quickly and reliably. MarkerLynx ${ }^{\mathrm{TM}}$ is a peak detection algorithm, where each mass number is analyzed separately in a search for peaks. The area of these peaks will be given an identity of $\mathrm{m} / \mathrm{z}$ and retention time and will be used as a fingerprint for each sample that is represented in relation to the other samples by principal component analysis. This software is repeatable and reliable analytical method when we should compare the informationrich spectroscopic data generated by UPLC/MS analysis from two or more group samples.

In this paper, the analgesic effects of two different extract of Shixiao San were investigated. Furthermore, UPLC-QTOF-MS coupled with Markerlynx ${ }^{\mathrm{TM}}$ were used to analyze the chemical components in different extracts of Shixiao San. This work demonstrated the potential of the UPLC/QTOFMS approach using Markerlynx ${ }^{\mathrm{TM}}$ combined with bioactivity evaluation for the characterization of the active constituents in Chinese herbal medicines 


\section{Results and Discussion}

\subsection{Effect of AESS and VESS on hot - plate test in mice}

In the hot-plate test, the results presented in table 1 showed that AESS (Aqueous Extract of Shixiao San) did not prolong the latency time at the tested dosage. As to VESS (Vinegary Extract of Shixiao San), after 1 hour of administration, the latency time was significantly increased both at its high and low dosage compared with the control group $(\mathrm{P}<0.05)$.

Table 1. Effect of AESS and VESS on hot - plate test in mice $(\bar{x} \pm s, n=10)^{\#}$.

\begin{tabular}{llllc}
\hline \multirow{2}{*}{ Group } & \multirow{2}{*}{ Dosage $(\mathbf{g} / \mathbf{k g})$} & \multicolumn{2}{c}{ Hot - plate latency (s) } \\
\cline { 3 - 5 } & & - & $\mathbf{1 ~ h}$ & $\mathbf{2 ~ h}$ \\
\hline \multicolumn{2}{l}{ Control group } & 0.025 & $15.84 \pm 3.81$ & $15.40 \pm 3.64$ \\
\multicolumn{2}{l}{ Pethidine group } & 4.90 & $32.37 \pm 6.56^{* *}$ & $16.45 \pm 4.11$ \\
AESS & High dose & 2.45 & $19.71 \pm 5.90$ & $17.48 \pm 6.74$ \\
group & Low dose & 10.06 & $20.40 \pm 3.73$ & $15.40 \pm 3.66$ \\
VESS & High dose & 5.03 & $20.21 \pm 3.76^{*}$ & $18.33 \pm 4.53$ \\
group & Low dose & $5.13 .13 \pm 5.49$ \\
\hline
\end{tabular}

The crude extracts were ensured to be equal in dose converting into crude plant materials, and the doses of crude plant materials for two extracts are equal; \# Values are expressed as mean \pm standard deviations $(\mathrm{n}=10)$; ** Level of significance relative to the control value $\mathrm{P}<0.01$; * Level of significance relative to the control value $\mathrm{P}<0.05$.

\subsection{Effect of AESS and VESS on acetic acid - induced writhing movements in mice}

Both AESS and VESS remarkably reduced the number of writhing times $(\mathrm{P}<0.01)$ induced by acetic acid. VESS decreased the numbers of writhing to $32.70 \pm 7.35$ at its low dose, and the inhibition ratio was $47.71 \%$, in comparison with that of AESS (20.50\%) (Table 2).

Table 2. Effect of AESS and VESS on acetic acid - induced writhing movements in mice $(\overline{\mathrm{x}} \pm \mathrm{s}, \mathrm{n}=10)^{\#}$.

\begin{tabular}{lllll}
\hline Group & & Dosage $(\mathbf{g} / \mathbf{k g})$ & Number of writhings & Inhibition $(\%)$ \\
\hline Control group & - & $56.10 \pm 5.86$ & - \\
Pethidine group & & 0.025 & $0.00^{* *}$ & 100.00 \\
\multirow{2}{*}{ AESS group } & High dose & 4.90 & $31.50 \pm 5.19^{* *}$ & 43.85 \\
& Low dose & 2.45 & $44.60 \pm 12.06^{*}$ & 20.50 \\
\multirow{2}{*}{ VESS group } & High dose & 10.06 & $29.11 \pm 7.01^{* *}$ & 48.10 \\
& Low dose & 5.03 & $32.70 \pm 7.35^{* *}$ & 47.71 \\
\hline
\end{tabular}

The crude extracts were ensured to be equal in dose converting into crude plant materials, and the doses of crude plant materials for two extracts are equal; \# Values are expressed as mean \pm standard deviations $(\mathrm{n}=10)$; ** Level of significance relative to the control value $\mathrm{P}<0.01$; * Level of significance relative to the control value $\mathrm{P}<0.05$. 


\subsection{Effects of AESS and VESS on dysmenorrhea mice models}

All of the test medications could remarkably reduce the writhing times $(\mathrm{P}<0.05)$ in a dymenorrhea mice model. The writhing inhibition rate of VESS low group is $60.68 \%$, which is superior to AESS low group (39.25\%) (as shown in Table 3). Meanwhile, the contents of $\mathrm{Ca}^{2+}$ and NO in the tissue homogenate of uterus were determined (Table 4). It was supposed that reduction of the writhing times by the tested medication was related to the decrease of $\mathrm{Ca}^{2+}$ and NO.

The previous three experiments showed that the bioactivity of VESS group was better than AESS group, which might be due to the chemical components changes during boiling with vinegar. So the chemical components in two different extracts are worthy of investigation.

Table 3. Effects of AESS and VESS on writhing of dysmenorrhea mice models $(\overline{\mathrm{x}} \pm \mathrm{s}, \mathrm{n}=10)^{\#}$.

\begin{tabular}{lllll}
\hline Group & Dose $\mathbf{( g / \mathbf { k g } )}$ & Number of writhings & Inhibition (\%) \\
\hline Normal group & - & 0 & 100 \\
Control group & - & $9.75 \pm 3.86$ & - \\
\multicolumn{2}{|l}{ Celecoxib group } & 0.0411 & $4.73 \pm 3.87^{*}$ & 51.52 \\
\multirow{2}{*}{ AESS group } & High dose & 4.90 & $5.00 \pm 3.02^{*}$ & 48.71 \\
& Low dose & 2.45 & $5.92 \pm 2.93^{*}$ & 39.25 \\
\multirow{2}{*}{ VESS group } & High dose & 10.06 & $4.00 \pm 2.66^{*}$ & 58.97 \\
& Low dose & 5.03 & $3.83 \pm 2.66^{*}$ & 60.68 \\
\hline
\end{tabular}

The crude extracts were ensured to be equal in dose converting into crude plant materials, and the doses of crude plant materials for two extracts are equal; \# Values are expressed as mean \pm standard deviations $(\mathrm{n}=10) ;$ * Level of significance relative to the control value $\mathrm{P}<0.05$.

Table 4. Effects of AESS and VESS on the content of $\mathrm{Ca}^{2+}$ and NO in the uterus tissue homogenate $(\overline{\mathrm{x}} \pm \mathrm{s}, \mathrm{n}=10)^{\#}$.

\begin{tabular}{|c|c|c|c|c|}
\hline \multicolumn{2}{|l|}{ Group } & Dose (g/kg) & $\mathrm{Ca}^{2+} / \mathrm{mmol}^{-g p r o t}{ }^{-1}$ & $\mathrm{NO} / \mu \mathrm{mol} \cdot \mathrm{gprot}^{-1}$ \\
\hline \multicolumn{2}{|l|}{ Normal group } & - & $0.08 \pm 0.07$ & $9.12 \pm 3.24$ \\
\hline \multicolumn{2}{|l|}{ Control group } & - & $0.44 \pm 0.21$ & $18.62 \pm 4.12$ \\
\hline \multicolumn{2}{|c|}{ Celecoxib group } & 0.0411 & $0.20 \pm 0.14$ & $18.73 \pm 7.25$ \\
\hline \multirow{2}{*}{ AESS group } & High dose & 4.90 & $0.13 \pm 0.07^{*}$ & $9.15 \pm 2.34^{*}$ \\
\hline & Low dose & 2.45 & $0.22 \pm 0.12^{*}$ & $11.98 \pm 3.44^{*}$ \\
\hline \multirow{2}{*}{ VESS group } & High dose & 10.06 & $0.11 \pm 0.18^{*}$ & $9.02 \pm 2.95^{*}$ \\
\hline & Low dose & 5.03 & $0.13 \pm 0.11^{*}$ & $10.46 \pm 3.05^{*}$ \\
\hline
\end{tabular}

The crude extracts were ensured to be equal in dose converting into crude plant materials, and the doses of crude plant materials for two extracts are equal; \# Values are expressed as mean \pm standard deviations $(\mathrm{n}=10) ;$ * Level of significance relative to the control value $\mathrm{P}<0.05$.

\subsection{UPLC - QTOF - MS analytic results}

Both the negative and positive ion modes were applied to analyze and identify the chemical components in the aqueous extract and vinegary extract of Shixiao San. The total current chromatograms with two ESI modes were shown in Figure 1. In order to eliminate the effects of 
vinegar, we analyzed the total ion chromatogram of vinegar in the same conditions. Twenty-four peaks were detected and eleven peaks were identified in two extracts, respectively. Through comparing the $t_{R}$ values, UV $\lambda_{\max }$ values, and the MS characteristics of the peaks with reference compounds and the literatures, the eleven identified compounds are listed as follows: quercetin-3-O-(2G- $\alpha$-1-rhamnosyl)rutinoside, isorhamnetin-3-O-rutinoside, typhaneoside, isorhamnetin-3-O-neohesperidoside, kaempferol-3-O-neohesperidoside, quercetin-3,3'-dimethyl ether, quercetin-3-O-neohesperidoside, kaempferol-3-O-glucoside, afzelin, $2 \alpha, 12 \beta$-dihydroxy-7,15-isopimardiene-18-oic-acid, and 2 $\alpha, 15 \mathrm{~S}, 16$ -trihydroxy-8(14)-isopimarene-18-oic-acid. The information about the analyzed and identified compounds is summarized in Table 5.

Figure 1. Total current chromatograms of AESS (A), VESS (B), Vinegar (C) in positive mode; and AESS (E), VESS (F), Vinegar (G) in negative mode.
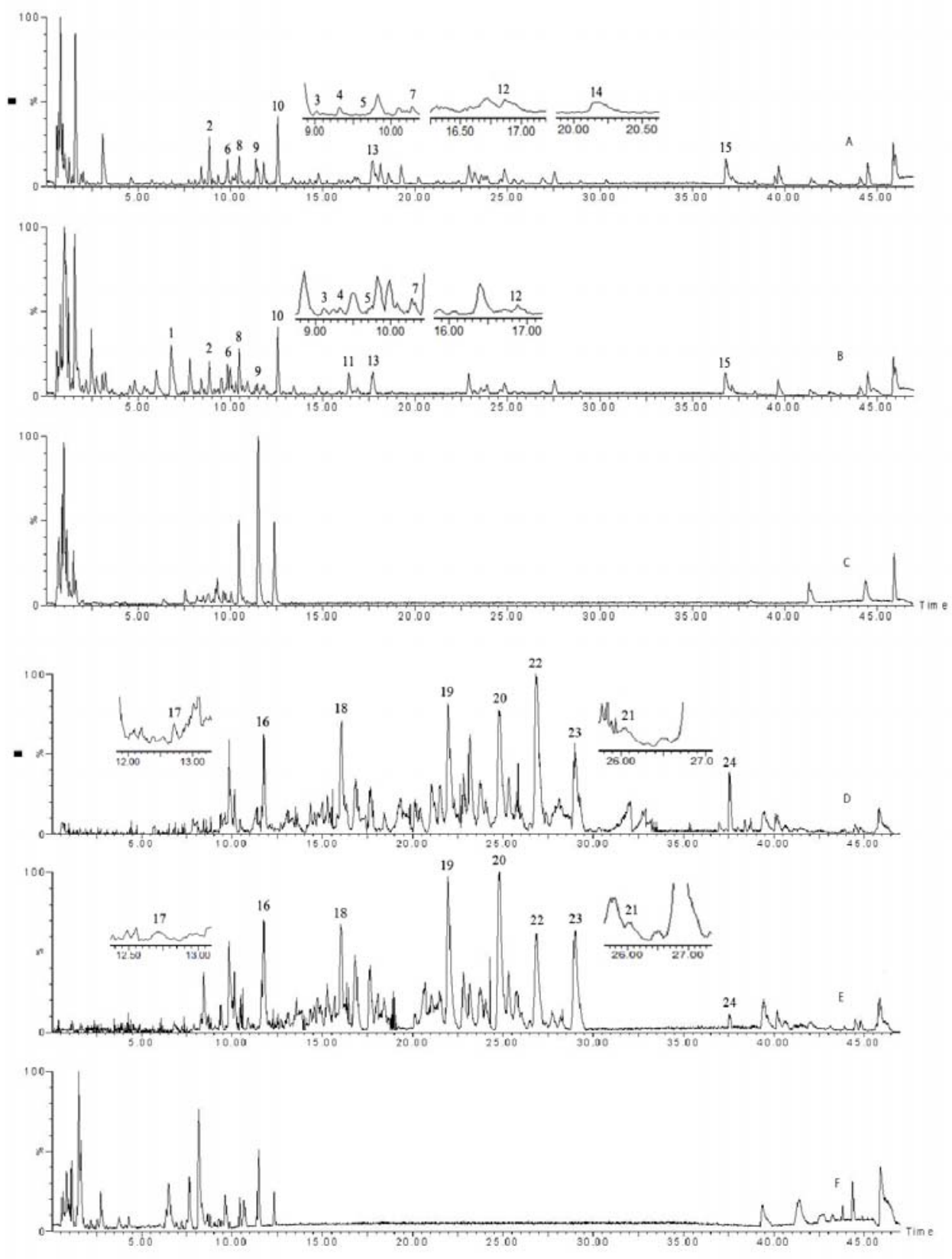
Table 5. UPLC - MS analytic results of AESS and VESS.

\begin{tabular}{|c|c|c|c|c|c|c|}
\hline NO. & $\begin{array}{l}\text { Retention } \\
\text { time (min) }\end{array}$ & Identification & $\begin{array}{c}\mathrm{UV} \\
\lambda_{\max }(\mathrm{nm})\end{array}$ & $\mathrm{ESI}^{+}$ & ESI $^{-}$ & $\begin{array}{c}\text { Source } \\
\text { herb }\end{array}$ \\
\hline 1 & 6.79 & unknown & 225 & - & $\begin{array}{l}231[\mathrm{M}+\mathrm{H}]^{+} \\
253[\mathrm{M}+\mathrm{Na}]^{+}\end{array}$ & $F T$ \\
\hline 2 & 8.83 & unknown & 228,272 & - & $\begin{array}{l}277[\mathrm{M}+\mathrm{H}]^{+} \\
299[\mathrm{M}+\mathrm{Na}]^{+}\end{array}$ & $P T / F T$ \\
\hline 3 & 9.11 & quercetin-3-O-(2G- $\alpha-1-$ rhamnosyl)-rutinoside & 255,353 & $\begin{array}{l}755[\mathrm{M}-\mathrm{H}]^{-}, 300[\mathrm{M}-\mathrm{H}-\text { rha-glu-rha }]^{-} \\
271[\mathrm{M}-2 \mathrm{H}-\text { rha-glu-rha-CO }]^{-}, 255[\mathrm{M}-2 \mathrm{H}-\text { rha-glu-rha-CO}]^{-}\end{array}$ & $757[\mathrm{M}+\mathrm{H}]^{+}$ & $P T$ \\
\hline 4 & 9.41 & quercetin-3-O-neohesperidoside & 255,355 & $\begin{array}{l}609[\mathrm{M}-\mathrm{H}]^{-}, 300[\mathrm{M}-\mathrm{H}-\text { rha-glu }]^{-}, 271[\mathrm{M}-2 \mathrm{H}-\text { rha-glu-CO }]^{-}, 255 \\
{\left[\mathrm{M}-2 \mathrm{H}-\mathrm{rha}-\mathrm{glu}-\mathrm{CO}_{2}\right]^{-}}\end{array}$ & $611[\mathrm{M}+\mathrm{H}]^{+}$ & $P T$ \\
\hline 5 & 9.70 & kaempferol-3-O- $\left(2^{\mathrm{G}}-\alpha-1-\right.$ rhamnosyl $)$-rutinoside & 265,348 & $\begin{array}{l}739[\mathrm{M}-\mathrm{H}]^{-}, 284[\mathrm{M}-\mathrm{H}-\text { rha-glu-rha }]^{-} \\
255[\mathrm{M}-2 \mathrm{H}-\text { rha-glu-rha-CO}]^{-}\end{array}$ & $\begin{array}{l}741[\mathrm{M}+\mathrm{H}]^{+} \\
763[\mathrm{M}+\mathrm{Na}]^{+}\end{array}$ & $P T$ \\
\hline 6 & 9.81 & isorhamnetin-3-O-( $2^{\mathrm{G}}-\alpha-1-$ rhamnosyl)-rutinoside & 254,354 & $\begin{array}{l}769[\mathrm{M}-\mathrm{H}]^{-}, 314{\text { [M-H-rha-glu-rha }]^{-}}, \\
285{\text { [M-2H-rha-glu-rha-CO }]^{-}}^{-}\end{array}$ & $771 \quad[\mathrm{M}+\mathrm{H}]^{+}$ & $P T$ \\
\hline 7 & 10.28 & kaempferol-3-O-neohesperidoside & 265,348 & $\begin{array}{l}593[\mathrm{M}-\mathrm{H}]^{-}, 284[\mathrm{M}-\mathrm{H}-\text { rha-glu-rha }]^{-}, \\
255[\mathrm{M}-2 \mathrm{H}-\text { rha-glu-rha-CO }]^{-}\end{array}$ & $\begin{array}{l}595[\mathrm{M}+\mathrm{H}]^{+} \\
617[\mathrm{M}+\mathrm{Na}]^{+}\end{array}$ & $P T$ \\
\hline 8 & 10.47 & isorhamnetin-3-O-neohesperidoside & 254,354 & $\begin{array}{l}623[\mathrm{M}-\mathrm{H}]^{-}, 314[\mathrm{M}-\mathrm{H}-\text { rha-glu-rha }]^{-} \\
285[\mathrm{M}-2 \mathrm{H}-\text { rha-glu-rha-CO }]^{-}\end{array}$ & $\begin{array}{l}625[\mathrm{M}+\mathrm{H}]^{+} \\
647[\mathrm{M}+\mathrm{Na}]^{+}\end{array}$ & $P T$ \\
\hline 9 & 11.24 & isorhamnetin-3-O-rutinoside & 254,354 & $\begin{array}{l}623[\mathrm{M}-\mathrm{H}]^{-}, 314{\text { [M-H-rha-glu-rha }]^{-}} \\
285[\mathrm{M}-2 \mathrm{H}-\text {-rha-glu-rha-CO }]^{-}\end{array}$ & $\begin{array}{l}625[\mathrm{M}+\mathrm{H}]^{+} \\
647[\mathrm{M}+\mathrm{Na}]^{+}\end{array}$ & $P T$ \\
\hline 10 & 12.55 & unknow & - & - & $\begin{array}{l}679[\mathrm{M}+\mathrm{H}]^{+} \\
701[\mathrm{M}+\mathrm{Na}]^{+}\end{array}$ & $P T / F T$ \\
\hline 11 & 16.42 & unknow & - & - & $\begin{array}{l}621 \quad[\mathrm{M}+\mathrm{H}]^{+} \\
643[\mathrm{M}+\mathrm{Na}]^{+}\end{array}$ & FT \\
\hline 12 & 16.87 & $2 \alpha, 12 \beta$-dihydroxy-7,15-isopimardiene-18-oic-acid & - & $333[\mathrm{M}-\mathrm{H}]^{-}$ & $335[\mathrm{M}+\mathrm{H}]^{+}$ & FT \\
\hline 13 & 17.71 & unknow & - & - & $621[\mathrm{M}+\mathrm{H}]^{+}$ & $F T$ \\
\hline 14 & 20.27 & $2 \alpha, 15 \mathrm{~S}, 16$-trihydroxy-8(14)-isopimarene-18-oic-acid & - & $351[\mathrm{M}-\mathrm{H}]^{-}, 387[\mathrm{M}+\mathrm{Cl}]^{-}$ & $353[\mathrm{M}+\mathrm{H}]^{+}$ & FT \\
\hline 15 & 36.87 & unknown & - & - & $274[\mathrm{M}+\mathrm{H}]^{+}$ & $F T$ \\
\hline 16 & 11.79 & unknown & - & $513[\mathrm{M}-\mathrm{H}]^{-}, 365,315$ & - & FT \\
\hline 17 & 12.71 & afzelin & 264 & $431[\mathrm{M}-\mathrm{H}]^{-}$ & $\begin{array}{l}433[\mathrm{M}+\mathrm{H}]^{+} \\
455[\mathrm{M}+\mathrm{Na}]^{+}\end{array}$ & FT \\
\hline 18 & 16.05 & unknown & - & $349[\mathrm{M}-\mathrm{H}]^{-}$ & - & $F T$ \\
\hline 19 & 22.00 & unknown & - & $331[\mathrm{M}-\mathrm{H}]^{-}, 119$ & - & $F T$ \\
\hline 20 & 24.93 & unknown & - & $413[\mathrm{M}-\mathrm{H}]-, 331,119$ & - & FT \\
\hline 21 & 26.07 & Quercetin-3,3'-dimethyl ether & 254,352 & $329[\mathrm{M}-\mathrm{H}]^{-}, 301[\mathrm{M}-\text { dimethyl ether }]^{-}$ & - & $P T$ \\
\hline 22 & 26.87 & unknown & - & $329[\mathrm{M}-\mathrm{H}]^{-}, 215$ & - & $F T$ \\
\hline 23 & 29.02 & unknown & - & $464[\mathrm{M}-\mathrm{H}]-, 411,329$ & - & FT \\
\hline 24 & 37.58 & unknown & - & $391[\mathrm{M}-\mathrm{H}]-, 325,243$ & - & $F T$ \\
\hline
\end{tabular}

PT: Pollen typha; FT: Faeces trogopterori; - : not confirmed. 


\subsection{Markerlynx ${ }^{T M}$ analytic results}

The principal components analysis was done using the Waters Markerlynx ${ }^{\mathrm{TM}}$ software. In this study, the sample of AESS, VESS and vinegar were injected five times each in two ESI modes. The results showed that the total current chromatograms of the three samples were significantly different, as shown in Figure 2. This can indicate that the chemical composition has changed after the process of boiling with vinegar. According to the intensity trends of fragment ions from three samples (Figure 3), the intensity of five flavonoids (typhaneoside, isorhamnetin-3-O-neohesperidoside, quercetin-3-Oneohesperidoside, kaempferol-3-O-neohesperidoside, quercetin-3-O-(2G- $\alpha$-L-rhamnosyl)-rutinoside) were higher in VESS than of in AESS. Moreover, the above-mentioned compounds' average values of peak area in VESS were list as follows: 238, 167, 123, 145, and 239. And the average values of peak area in AESS were 148, 115, 93, 103, and 174. So the contents of the detected five flavonoids were increased after the process of boiling in vinegar.

Figure 2. The scores plot obtained from Markerlynx ${ }^{\mathrm{TM}}$ analytical results in positive mode (A) and negative mode (B).
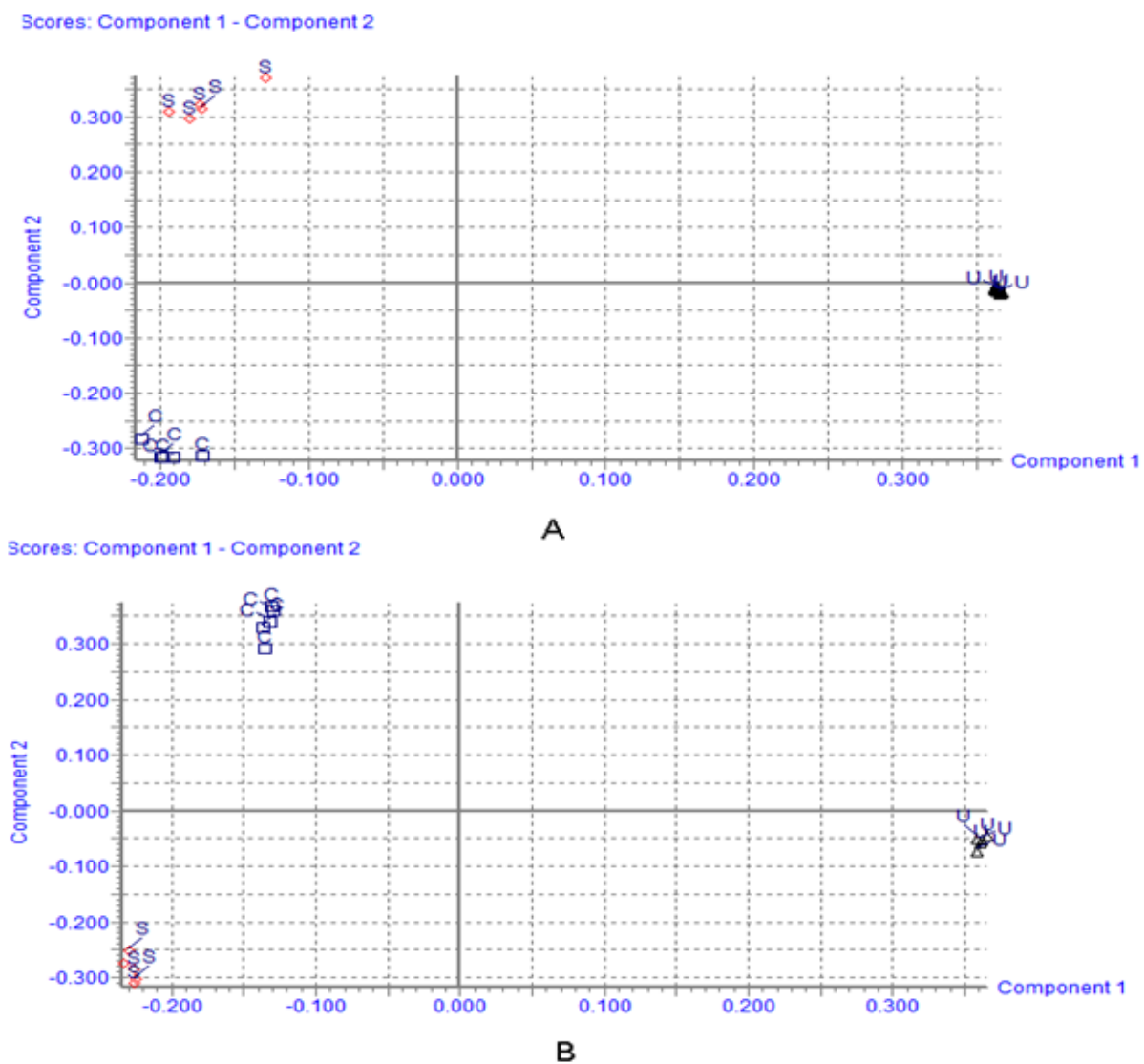

The result is based on three samples of AESS $(\diamond)$, VESS ( $\square)$ and Vinegar $(\Delta)$, respectively. 
Figure 3. The variation trends of fragment ions intensity of identified compounds from three samples of AESS $(\diamond)$, VESS $(\square)$ and Vinegar $(\Delta)$. (a) typhaneoside. (b) quercetin-3$O$-(2G- $\alpha$-L-rhamnosyl)-rutinoside. (c) isorhamnetin-3-O-neohesperidoside. (d) quercetin-3$O$-neohesperidoside. (e) kaempferol-3-O-neohesperidoside.
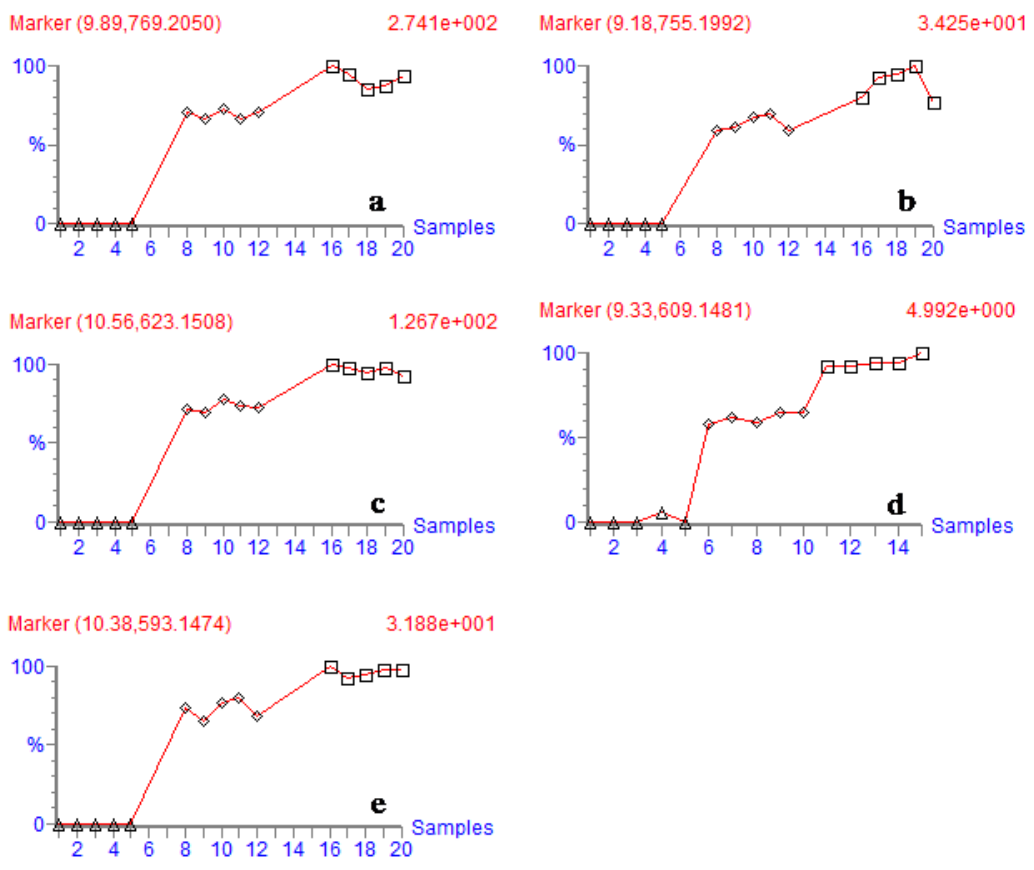

These trends can indicate the contents of the corresponding compounds in the three samples. The fragment ions here are the molecular ion $\left(\mathrm{M}^{-}\right)$peaks (x axis: the number of sample injection; $y$ axis: fragment ions abundances).

\subsection{Discussion}

In this work, we first developed a new method of using bioactivity evaluation followed by UPLC/QTOFMS and Markerlynx ${ }^{\mathrm{TM}}$ to quickly determine and analyze the information-rich data for characterizing the active components in TCMs. Moreover, this work demonstrated that this potential approach for the characterization of the active constituents in TCMs is effective and feasible.

It was considered in Traditional Chinese Medicine that the TCM formula involves the compatibility of two or more medications, that could interact and increase the effect of any single drug. In the present study, the compatibility of two medications (Puhuang - Wulingzhi) resulted in remarkable biological effects. After the process of boiling with vinegar, the effects were promoted. The results in this paper suggested that the process of boiling in vinegar might improve dissolution of active components and enhance the analgesic activity.

The hot-plate test and acetic acid-induced writhing test reflected central and peripheral nociceptive model respectively. Dysmenorrhea mice model were adopted to evaluate the analgesic activity of Shixiao San. The results showed that the analgesic effects of vinegary extract were better than those of the aqueous extract. Shixiao San is frequently used to treat primary dysmenorrheal in the clinic in China [2]. The mechanism of primary dysmenorrhea may be related to intracellular free calcium and the levels of prostaglandin $\mathrm{F}_{2 \alpha}$. In addition, $\mathrm{NO}$ is the active factor involved in the stimulation of COX- 
2 enzyme [10-12]. This study showed that Shixiao San extracts significantly inhibited the writhing response on the primary dysmenorrhea mice, and reduced the content of $\mathrm{Ca}^{2+}$ and NO in uterine tissue. Therefore these data implied that the mechanisms of inhibitory activity on myometrial contractility of VESS or AESS were related with the lower of $\mathrm{Ca}^{2+}$ and NO contents in the smooth muscle cell.

It was known that multiple constituents are responsible for their bioactivities of TCMs [13], however, due to the complexity of chemical compositions, the responsible bioactive compounds are difficult to confirm. Therefore, it is important and urgent to carry out chemical and pharmacological correlated studies on TCMs to elucidate the biologically active compounds and their therapeutic mechanisms [14]. In this paper, the method combining pharmacological experiments and UPLC/QTOFMS and Markerlynx ${ }^{\mathrm{TM}}$ proved effective for the fast characterization of the active constituents in different preparations of Shixiao San. This method is a simple, repeatable and reliable testing technique for comparing the information-rich spectroscopic data generated by UPLC/MS analysis from two or more group samples. It can reveal the difference of various samples much more quickly and exactly than traditional approaches like HPLC-DAD.

The data of chemical constituents' changes between aqueous extract and vinegar extract showed that the total current chromatograms of two samples were different significantly, and the ion intensity of five flavonoid compounds were also different through the UPLC/QTOFMS coupled with Markerlyn $x^{\mathrm{TM}}$ method. It can be inferred that the processing of vinegar may promote the dissolution of flavonoids by intermolecular hydrogen bonds, so the changing components in different extracts may result in different bioactivities, and these components (flavones glycosides) may be the active constituents. Moreover, these flavonoids possessed multiple activities as antiflammatory, analgesic, immunosuppressive compounds [15-17]. Modern pharmacological studies show that kaempferol-3-Oneohesperidoside and quercetin-3-O-neohesperidoside can inhibit the activity of nitric oxide produced by activated macrophages [18] and kaempferol glycosides may account for the renowned medicinal use of Sedum dendroideum against pain and inflammatory problems [19].

\section{Experimental}

\subsection{Materials}

The raw materials of Pollen Typha and Faeces Trogopterori were purchased from Jiansu (Yixing) and Hebei (Anguo), respectively. All the crude herbs were identified by the corresponding author. Voucher specimens (No. NJUTCM - 20090118 20090119) were deposited in Jiangsu Key Laboratory for TCM Formulae Research, Nanjing University of Chinese Medicine, China.

\subsection{Chemicals and reagents}

Estradiol benzoate injection (Tianjin Jinyao Amino Acid Pharmaceutical Co., Ltd., Tianjin, China NO. 0705161), oxytocin injection (Shanghai First Biochemical Pharmaceutical Co., Ltd., Shanghai, China, NO. 071116), Celecoxib capsules (Pfizer Pharmaceuticals LLC, NO. BK081004), $\mathrm{Ca}^{2+}$ test kit and NO test kit (Nanjing Jiancheng Bioengineering Institute Co., Ltd., Nanjing, China, NO. 20081204; NO. 20081204), pethidine injection (Qinghai Pharmaceutical Co., Ltd., Qinghai, China, No. 050310), Vinegar (Jiangsu Hengshun Vinegar Co., Ltd., Jiangsu, Zhenjiang, China). 
Acetonitrile was HPLC-grade from Merck (Darmstadt, Germany) and deionized water was purified by an EPED super purification system (Eped, Nanjing, China). The reference compounds typhaneoside, isohamnetin-3-O-neohespeidoside, and isohamnetin-3-O-rutinoside were purchased from the National Institute for the Control of Pharmaceutical and Biological Products (Beijing, China), quercetin-3-O-(2G- $\alpha$-L-rhamnosyl)-rutinoside, afzelin, kaempferol-3-O-glucoside, kaempferol-3-Oneohesperidoside, quercetin-3,3'-dimethyl ether, quercetin-3-O-neohesperidoside, 2,12-dihydroxy7,15-isopimardiene-18-oic-acid and 2,15S,16- trihydroxy-8(14)-isopimarene-18-oic-acid were isolated by our laboratory. Other reagents solutions were analytical grade (Sinopharm Chemical Reagent Co., Ltd., Shanghai, China).

\subsection{Animals}

All experiments were performed with female ICR mice, weighing 18-22 g, obtained from the experimental animal center of China Pharmaceutical University. They were kept in plastic cages at $22 \pm 2{ }^{\circ} \mathrm{C}$ with free access to pellet food and water and on a $12 \mathrm{~h} \mathrm{light/dark} \mathrm{cycle.} \mathrm{Animal} \mathrm{welfare} \mathrm{and}$ experimental procedures were carried out in accordance with the guide for the care and use of laboratory animals (National Research Council of USA, 1996) and related ethical regulations of our university. Groups each with 10 animals were used in all tests.

\subsection{Instrumentation}

Chromatographic experiments were performed on a Waters ACQUITYTM UPLC ${ }^{\text {TM }}$ system connected to Synapt ${ }^{\mathrm{TM}}$ Q-TOF Premier equipped with an electrospray ionization source, which was used in positive or negative mode(Waters Corp., Milford, MA, USA). UPLC separation was achieved using an ACQUITY UPLC ${ }^{\text {TM }}$ BEH $\mathrm{C}_{18}$ column $(100 \times 2.1 \mathrm{~mm}, 1.7 \mu \mathrm{m})$. Data acquisition and processing were performed using MassLynx 4.1 and Markerlynx 4.1 (Waters Corp., Milford, MA, USA). MarkerLynx ${ }^{\mathrm{TM}}$ Application Manager is a software package from Waters (Mass., USA) for peak detection. Automatic Microplate Reader (Thermo, USA); TGL - 16C High Speed Centrifuge (Shanghai Anting Scientific Device Co., Ltd., Shanghai, China); AL204 Electronic Balance (Mettler Toledo); YLS - 6B Hot Plate (Beijing Gene \& I Scientific Ltd., Beijing, China).

\subsection{Preparation of samples}

\subsubsection{Preparation of aqueous extract of Shixiao San (AESS)}

Puhuang and Wulingzhi (500 g respectively) were extracted twice $(1 \mathrm{~h} /$ time $)$ under reflux with $10,000 \mathrm{~mL}$ water $(\mathrm{pH}=7.5)$. The combined extract was concentrated under reduced pressure to give the dried extract (yield 11.9\%) as the extract sample 1.

\subsubsection{Preparation of vinegary extract of Shixiao San (VESS)}

Puhuang and Wulingzhi (500 g respectively) were boiled with 1,000 $\mathrm{mL}$ vinegar $(\mathrm{pH}=3.5)$ for about $2.5 \mathrm{~h}$ until dryness, and then extracted twice $(1 \mathrm{~h} /$ time $)$ under reflux with $10,000 \mathrm{~mL}$ water. The 
combined extract was concentrated under reduced pressure to give the dried extract (yield 25.7\%) as the extract sample 2 .

\subsection{Hot - plate test in mice}

Experiments were carried out according to the previously described method [20]. Mice were pretreated with test medication, respectively. Animals were habituated twice to the hot-plate in advance. For testing, mice were placed on hot-plate maintained at $55 \pm 5{ }^{\circ} \mathrm{C}$. The interval of time between occurrence of either a hind paw licking or a jump off the surface was recorded as the hotplate latency. Mice with baseline latencies of $<5 \mathrm{~s}$ or $>30 \mathrm{~s}$ were eliminated from the study. Hot-plate latencies were determined at $60 \mathrm{~min}$ and $120 \mathrm{~min}$ after oral administration of the tested medication.

ICR mice were divided into six groups, and the control group was administrated isovolumetric distilled water. The typical dose of crude plant material of Shixiao San for a person was about $20 \mathrm{~g} /$ day (Puhuang and Wulingzhi was $10 \mathrm{~g}$ respectively) according to folk remedies. After the preliminary experiments, two effective dosages were selected, and the crude extracts were adjusted to be equal in dose converting into crude plant materials. The dosage of the test medication was AESS group i. $\mathrm{g}$. $4.9 \mathrm{~g} / \mathrm{kg}$, and $2.45 \mathrm{~g} / \mathrm{kg}$ of crude extracts, VESS group i. g. $10.06 \mathrm{~g} / \mathrm{kg}$, and $5.03 \mathrm{~g} / \mathrm{kg}$ of crude extracts, and the mice were administered for seven days. The mice of the positive group were injected pethidine before being placed on the hot-plate.

\subsection{Acetic acid - induced writhing response in mice}

The acetic acid - induced writhing model was basically established as described in the literatures $[21,22]$. ICR mice were pretreated with test medication for 7 days, $1 \mathrm{~h}$ before the injection of $0.6 \%$ aqueous solution of acetic acid $(10 \mathrm{~mL} / \mathrm{kg}$, i. p.) last time. Each mouse was placed in a transparent observation box and the number of writhings was counted for $20 \mathrm{~min}$ after the acetic acid administration. The number of writhes in each treated group was compared with the control group. The inhibition rate of writhing [(control mean - test mean $) /$ control mean] $\times 100$ was calculated. ICR mice were divided into 6six groups, and control group was administrated isovolumetric distilled water. The dosage of the test medication was the same as previous experiments, and the mice were administered for seven days. The mice of positive group were injected pethidine.

\subsection{Dysmenorrhea mice model preparation}

According to the reported method [23,24], we used estradiol benzoate and oxytocin to make the dysmenorrhea mice model. Estradiol benzoate $(0.01 \mathrm{~g} / \mathrm{kg})$ was administrated by subcutaneous injection for 6 days, and on the seventh day, oxytocin $(10 \mathrm{~mL} / \mathrm{kg})$ was administrated by peritoneal injection 60 min after the final administration. ICR mice were divided into six groups, and the normal group was administrated isovolumetric distilled water, the control group was treated with estradiol benzoate and oxytocin and administrated distilled water. The dosage of the test medication was the same as previous experiments. The celecoxib group which was positive group was treated with estradiol benzoate and oxytocin and i. g. $0.0411 \mathrm{~g} / \mathrm{kg}$ of what. From the fifth day, all the test medications were administrated 
for three days. Record the number of writhings occurring from 5 to $20 \mathrm{~min}$, and determine the content of $\mathrm{Ca}^{2+}$ and $\mathrm{NO}$ in the homogenate of uterus according to specification of kits.

\subsection{Statistical analysis}

The results were expressed as mean \pm S.D. and evaluated with one - way ANOVA following by Dunnett's multiple comparisons test. A $p$ value of less than 0.05 was considered statistically significant and $p$ less than 0.01 being very significant.

\subsection{UPLC - QTOF - MS analytical conditions}

The mobile phase was composed of A (acetonitrile), B (0.5\% aqueous acetic acid, v/v) with a linear gradient elution: 0 5min, A: 5\%; 5 8 $\mathrm{min}, \mathrm{A}: 5 \% \sim 15 \% ; 8 \sim 11 \mathrm{~min}, \mathrm{~A}: 15 \% \sim 20 \% ; 11 \sim 19 \mathrm{~min}$, A: 20\% 25\%; 19 35 min, A: 25\% 35\%; 35 43 min, A: 35\% 80\%; 43 45 min, A: 80\%; 45 46 min, A: $80 \% \sim 5 \%$; 46 47min, A: 5\%. The flow rate of the mobile phase was $0.4 \mathrm{~mL} / \mathrm{min}$, and the column temperature was maintained at $30^{\circ} \mathrm{C}$. The ionization source conditions were as follows: capillary voltage of $1.7 \mathrm{kV}$, source temperature of $100^{\circ} \mathrm{C}$ and desolvation temperature of $250{ }^{\circ} \mathrm{C}$. The sampling cone voltage was set at $40 \mathrm{~V}$, extraction cone at $0.8 \mathrm{~V}$, trap collision energy 6.0 , transfer collision energy 4.0, trap gas flow were $1.50 \mathrm{~mL} / \mathrm{min}$, ion energy at $1.0 \mathrm{~V}$, collision energy at $4.0 \mathrm{~V}$. Nitrogen and argon were used as cone and collision gases, respectively. The cone and desolvation gas flow were 50 and $600 \mathrm{~L} / \mathrm{h}$, respectively. The scan time of $0.6 \mathrm{~s}$ and with interval scan time of $0.02 \mathrm{~s}$ was used throughout and with collision energy of $6 \mathrm{eV}$. The mass spectrometric data was collected from $\mathrm{m} / \mathrm{z} 100$ to 1000 in positive and negative ion in centroid mode. The MS/MS experiments were performed at variable collision energies $(6-40 \mathrm{eV})$ which were optimized for each individual compound.

\subsection{MarkerLynx ${ }^{T M}$}

Data processing were performed using Markerlynx ${ }^{\mathrm{TM}}$ software which with the help of multivariate statistical analysis, revealed the possible changed components, and the identities of which can be extensively determined with online UV and MS information. MarkerLynx ${ }^{\mathrm{TM}}$ operates in two steps: firstly, peak detection is performed using ApexPeak Track. The peaks from different samples were aligned so that the same peaks (RT, $\mathrm{m} / \mathrm{z}$ ), i.e., most probably the same compound, were found in the same row for all samples. In this process, sample name and ion intensity were analyzed by supervised orthogonal partial least squared discriminate analysis (OPLS-DA) using the MarkerLynx ${ }^{\mathrm{TM}}$ software; and then, all of the results, including the Markers table and PCA Scores and loading plots are viewed via the MarkerLynx browser. The ion intensities for each detected peak were normalized against the sum of the peak intensities within that sample using MarkerLynx ${ }^{\mathrm{TM}}$. Ions of different samples were considered to be the same ion when they demonstrated the same $t_{R}$ (tolerance of $0.01 \mathrm{~min}$ ) and $\mathrm{m} / \mathrm{s}$ value (tolerance of $0.01 \mathrm{Da}$ ). If a peak was not detected in a sample, the ion intensity was documented as zero in the table.

The original data were processed using the following parameters: initial retention time 0 min, final retention time $40 \mathrm{~min}$, mass tolerance $0.02 \mathrm{Da}$, mass window $0.02 \mathrm{Da}$, retention time window $0.1 \mathrm{~min}$, 
noise elimination level 6. PCA reduces a complex data set to a $2 \mathrm{D}$ scores plot showing the intrinsic patterns in the data and a 2D loading plot that highlights the quantities responsible for the patterns.

\section{Conclusion}

In this paper, the results proved that the components changes of Shixiao San triggered by different preparations generated different bioactivities. The advantages of the proposed method over the conventional chromatographic fingerprints and marker compounds are the speed and reliability to determine the potential active components in different extracts, such as VESS and AESS. The data in this article suggested that the new method could provide thoughts and techniques for revealing the possible interactions between TCMs.

\section{Acknowledgements}

This work was supported by a National Natural Science Foundation of China (No. 30973885), a 2006 Key Research Project in Basic Science of Jiangsu College and University (NO. 06KJA36022), and a 2006 Training Program of Scientific and Technological Innovation Team for "Qinglan Project" of Jiangsu College and University. And this study also was granted by Natural Science Foundation for Young Scholars of Nanjing University of Chinese Medicine (NO. 09XZR15) and Scientific and Technological Program of Jiangsu Traditional Chinese Medicine (NO. LB09016).

\section{References and Notes}

1. Huang, Y.J.; Wu, X.Y. Research progression and clinical application of Shixiao San. Chin. Trad. Plant Med. 1995, 17, 40-41.

2. Zhou, W.; Su, S.L.; Duan, J.A.; Tao, W.W. The association analysis of shixiaosan's traditional utility and modern research. Chin. Trad. Plant Med. 2009, 31, 1058-1061.

3. Li, Q.; Sun, J.N.; Xia, X.H.; Xiao, H.B.; Song, C.Y.; Lin, Q.; Li, X.Z. A Pharmacodynamic study on shixiaosan and its extracts. J. Beijing Univ. TCM 1999, 22, 48-49.

4. Zhang, P.; Xia, X.H.; Li, Q. The effects of Anti - thrombosis and thrombolysis of diferent solvent extract of shixiaosan. China Pharm. 2003, 12, 44-45.

5. Li, Q.; Lu, Y.R.; Lu, X.Z. The study on progression of Faeces Trogopterori. Zhongguo Zhong Yao Za Zhi 1998, 23, 570-573.

6. Zhang, Q.Y.; Mao, J.H.; Liu, P.; Huang, Q.H.; Lu, J.; Xie, Y.Y.; Weng, L.; Zhang, Y.; Chen, Q.; Chen, S.J.; Chen, Z. A systems biology understanding of the synergistic effects of arsenic sulfide and imatinib in BCR/ABL-associated leukemia. Proc. Nat. Acad. Sci. USA 2009, 106, 3378-3383.

7. Li, S.L.; Song, J.Z.; Qiao, C.F.; Zhou, Y.; Xu, H.X. UPLC-PDA-TOFMS based chemical profiling approach to rapidly evaluate chemical consistency between traditional and dispensing granule decoctions of traditional medicine combinatorial formulae. J. Pharm. Biomed. Anal. 2010, 52, 468-478.

8. Normile, D. ASIAN MEDICINE: The New Face of Traditional Chinese Medicine. Science 2003, 299, 188-190.

9. Su, S.L.; Guo, J.M.; Duan, J.A.; Wang, T.J.; Qian, D.W.; Shang, E.X.; Tang, Y.P. Ultraperformance liquid chromatography-tandem mass spectrometry analysis of the bioactive 
components and their metabolites of Shaofu Zhuyu decoction active extract in rat plasma. $J$. Chromatogr. B. 2010, 878, 355-362.

10. Salvemini, D.; Misko, T.P.; Masferrer, J.L.; Seibert, K.; Currie, M.G.; Needleman, P. Nitric oxide activates cyclooxygenase enzymes. Proc. Nat. Acad. Sci. USA 1993, 90, 7240-7244.

11. Dawood, M.Y. Primary dysmenorrhea: advances in pathogenesis and management. Obstet. Gynecol. 2006, 108, 428-441.

12. Raine-Fenning, N. Dysmenorrhoea. Obstet. Gynaecol. Reprod. Med. 2008, 18, 294-299.

13. Li, T.; Roy, R. Studying traditional Chinese Medicine. Science 2003, 300, 740-741.

14. Su, S.L.; Hua, Y.Q.; Duan, J.A.; Shang, E.X.; Tang, Y.P.; Bao, X.J.; Lu, Y.; Ding, A.W. Hypothesis of active components in volatile oil from a Chinese herb formulation, Shao-Fu-ZhuYu decoction', using GC-MS and chemometrics. J. Seperation Sci. 2008, 31, 1085-1091

15. Qin, F.; Sun, H.X. Immunosuppressive activity of Pollen Typhae ethanol extract on the immune responses in mice. J. Ethnopharmacol. 2005, 102, 424-429.

16. Wang, H.B.; Wang, Z.Y. A study of the analgesic effect and acute toxicity of the extract of the Traditional Chinese Medicine Pollen Typhae. Her. Med. 2006, 25, 278-279.

17. Park, W.H.; Kim, C.H.; Lee, Y.C.; Kim, C.H. Anti-inflammatory effects of a traditional Korean herbal formulation, Silsosangami, consisting of seven medicinal herbs: effect on hemolysis, neutrophil function, and gene expressions of iNOS and COX-2. Vasc. Pharmacol. 2004, 42, 7-15.

18. da Silva, B.P.; Bernardo, R.R.; Parente, J.P. Flavonol glycosides from Costus spicatus. Phytochemistry 2000, 53, 87-92.

19. De Melo, G.O.; Malvar, D.D.; Vanderlinde, F.A.; Rocha, F.F.; Pires, P.A.; Costa, E.A.; de Matos, L.G.; Kaiser, C.R.; Costa, S.S. Antinociceptive and anti-inflammatory kaempferol kaempferol glycosides from Sedum dendroideum. J. Ethnopharmacol. 2009, 124, 228-232.

20. Adzu, B.; Amos, S.; Kapu, S.D.; Gamaniel, K.S. Anti - inflammatory and anti - nociceptive effects of Sphaeranthus senegalensis. J. Ethnopharmacol. 2003, 84, 169-173.

21. Fukawa, K.; Kawano, O.; Hibi, M.; Misaki, N.; Ohba, S.; Hatanaka, Y. A method for evaluating analgesic agents in rats. J. Pharmacol. Methods 1980, 4, 251-259.

22. Ribeiro, R.A.; Vale, M.L.; Thomazzi, S.M.; Paschoalato, A.B.; Poole, S; Ferreira, S.H.; Cunha, F.Q. Involvement of resident macrophages and mast cells in the writhing nociceptive response induced by zymosan and acetic acid in mice. Eur. J. Pharmacol. 2000, 387, 111-118.

23. Sun, H.Y.; Cao, Y.X.; Liu, J.; Gao, J.W.; Ma, M. The establishment of the dysmenorrhea model in mice. Chin. Pharmacol. Bull. 2002, 18, 233-235.

24. Ostad, S.N.; Soodi, M.; Shariffzadeh, M.; Khorshidi, N.; Marzban, H. The effect of fennel essential oil on uterine contraction as a model for dysmenorrhea, pharmacology and toxicology study. J. Ethnopharmacol. 2001, 76, 299-304.

Sample Availability: Samples of the compounds quercetin-3-O-(2G- $\alpha-\mathrm{L}-$ rhamnosyl)-rutinoside, afzelin, kaempferol-3-O-glucoside, kaempferol-3-O-neohesperidoside, quercetin-3,3'-dimethyl ether, quercetin-3-O-neohesperidoside, 2,12-dihydroxy-7,15-isopimardiene-18-oic-acid and 2,15S,16-trihydroxy-8(14)-isopimarene-18-oic-acid are available from the authors.

(C) 2010 by the authors; licensee MDPI, Basel, Switzerland. This article is an Open Access article distributed under the terms and conditions of the Creative Commons Attribution license (http://creativecommons.org/licenses/by/3.0/). 